COMMUNICATIONS IN

ANALYSIS AND GEOMETRY

Volume 14, Number 4, 703-723, 2006

\title{
Liouville-type properties for embedded minimal surfaces
}

\author{
William H. Meeks III ${ }^{1}$, Joaquín Pérez and Antonio Ros
}

In this paper, we study conformal properties of complete embedded minimal surfaces in flat three-manifolds. These properties include recurrence, transience and the existence/nonexistence of nonconstant bounded and/or positive harmonic functions. We also apply these results to study the question of existence of complete embedded minimal surfaces which are $a$-stable for some $a>0$.

\section{Introduction}

The classical Liouville theorem for the plane asserts that every positive harmonic function on the complex plane is constant. The upper halfplane $\mathbb{H}=\left\{(x, y) \in \mathbb{R}^{2} \mid y>0\right\}$ does not satisfy the same property. The existence or nonexistence of nonconstant positive harmonic functions can be viewed as a tool for understanding the so called type problem of classifying open Riemann surfaces. There are related properties useful for tackling this problem on a noncompact Riemann surface $M$ without boundary, among which we emphasize the following ones:

1. $M$ admits a nonconstant positive superharmonic function (equivalently, $M$ is transient for Brownian motion).

2. $M$ admits a nonconstant positive harmonic function.

3. $M$ admits a nonconstant bounded harmonic function.

A noncompact Riemann surface $M$ without boundary is said to be recurrent for Brownian motion if it does not satisfy property (1) (see, e.g., Grigor'yan [6] for a detailed study of these properties and for general properties of Brownian motion on manifolds). Clearly $(3) \Rightarrow(2) \Rightarrow(1)$.

\footnotetext{
${ }^{1}$ This material is based upon work for the NSF under Award No. DMS0405836. Any opinions, findings and conclusions or recommendations expressed in this publication are those of the authors and do not necessarily reflect the views of the NSF.
} 
These three conditions are known to be equivalent if $M$ has finite genus, or even almost-finite genus (see [23] for the definition and result).

The Liouville conjecture for properly embedded minimal surfaces states that positive harmonic functions on properly embedded minimal surfaces in $\mathbb{R}^{3}$ are constant [11]. In relation with the above properties and the Liouville conjecture, we note that in a previous paper [14], we proved that every properly embedded minimal surface with finite genus in $\mathbb{R}^{3}$ is recurrent for Brownian motion, and so, the Liouville conjecture holds for these surfaces. We conjectured in [13] that properly embedded minimal surfaces of almost-finite genus in complete flat three-manifolds are recurrent. Note that embeddedness in this conjecture is essential since there exists a properly immersed minimal disk in $\mathbb{R}^{3}[20]$. Since every complete Riemann manifold with quadratic volume growth is recurrent [6], each of the classical Scherk singly periodic minimal surfaces in $\mathbb{R}^{3}$ is recurrent. Another class of properly embedded minimal surfaces in $\mathbb{R}^{3}$ which are known to be recurrent are those with two limit ends [2].

Recent research demonstrates that certain complete embedded minimal surfaces in a complete flat three-manifold $N$ are in fact properly embedded. For example, the minimal lamination closure theorem [16] implies that complete embedded nonflat minimal surfaces $M \subset N$ are properly embedded provided that the injectivity radius of $M$ is positive, for instance if $M$ has bounded curvature. Since complete embedded minimal surfaces of finite topology in $N$ always have positive injectivity radius, one recovers the result of Colding and Minicozzi [1] that complete embedded minimal surfaces of finite topology in $\mathbb{R}^{3}$ are proper. More generally, we proved [13] that complete embedded minimal surfaces in $\mathbb{R}^{3}$ with finite genus and a countable number of ends are properly embedded. Because of these results, we will always assume properness for the minimal surfaces in the proofs of our results.

In this paper, we shall determine which of the above three properties are satisfied by a complete embedded minimal surface $M \subset \mathbb{R}^{3}$, in terms of its topology and geometry. Let $G$ be a group of orientation preserving isometries acting properly and discontinuously on $\mathbb{R}^{3}$, which leaves $M$ invariant. Since the properties we are interested in - recurrence, transience and existence of unbounded positive harmonic functions - hold simultaneously for the base and the total space of a finitely sheeted covering space, and bounded harmonic functions lift to arbitrary covers, we will assume that $G$ is either \{identity\} or it is generated by two or three independent translations, or by a translation or screw motion symmetry. We will say that $M$ is $k$-periodic if $G$ has rank $k=0,1,2,3$. Next, we summarize the main results. 
Theorem 1.1. A complete embedded 1-periodic minimal surface with bounded curvature in $\mathbb{R}^{3}$ does not admit nonconstant bounded harmonic functions, and its quotient surface is recurrent.

Theorem 1.2. A properly immersed 2-periodic minimal surface in $\mathbb{R}^{3}$ does not admit nonconstant bounded harmonic functions, and its quotient surface is recurrent.

Theorem 1.3. Let $M \subset \mathbb{R}^{3}$ be a nonflat $k$-periodic complete embedded minimal surface such that $M / G \subset \mathbb{R}^{3} / G$ has finite topology. Then, $M$ is recurrent if and only if $k=0$ or 1 .

Theorem 1.4. Let $M \subset \mathbb{R}^{3}$ be a complete embedded minimal surface such that $M / G$ has finite topology. Then, every positive harmonic function on $M$ is constant.

The study of complete stable minimal surfaces in complete flat threemanifolds is closely related to some of the above conformal questions, as we next explain. Let $M \subset N$ be a minimal surface in a complete flat threemanifold $N$. Given $a>0$, we say that $M$ is a-stable if for any compactly supported smooth function $u \in C_{0}^{\infty}(M)$, we have

$$
\int_{M}\left(|\nabla u|^{2}+a K u^{2}\right) d A \geq 0
$$

where $\nabla u$ stands for the gradient of $u$ and $K, d A$ are the Gaussian curvature and the area element on $M$, respectively (the usual stability condition for the area functional corresponds to the case $a=2$ ). The key connection between $a$-stability and transience is based on the following fact.

Proposition 1.5. If $M$ is a complete nonflat a-stable minimal surface in a complete flat three-manifold $N$, then $M$ is transient for Brownian motion.

Do Carmo and Peng [3], Fischer-Colbrie and Schoen [5] and Pogorelov [21] proved independently that if $M \subset \mathbb{R}^{3}$ is a complete, orientable $a$-stable minimal surface, for $a \geq 1$, then $M$ is a plane. This result was improved by Kawai [7] to $a>1 / 4$, see also Ros [22]. In this article, we will use Proposition 1.5 to obtain flatness of complete embedded $a$-stable minimal surfaces in complete flat three-manifolds, under the additional topological assumptions of finite genus. 
Theorem 1.6. Let $N$ be a complete flat three-manifold and let $a>0$. Then, any complete embedded a-stable minimal surface $M \subset N$ with finite genus is totally geodesic (flat).

Based on the above results, we make the following conjecture.

Conjecture 1.7 (Meeks, Pérez, Ros). A complete embedded a-stable minimal surface in a complete flat three-manifold is totally geodesic (flat).

We will apply our recent Local Picture Theorem on the Scale of Curvature and Dynamics Theorem [12] for properly embedded minimal surfaces to reduce the solution of Conjecture 1.7 to the particular case given in the next theorem.

Theorem 1.8. If there exists a complete embedded nonflat a-stable minimal surface in a complete flat three-manifold, then there exists a properly embedded nonflat a-stable minimal surface $\Sigma \subset \mathbb{R}^{3}$ which has infinite genus, bounded curvature and is quasi-dilation-periodic. On the other hand, no such $\Sigma$ can be 1-periodic.

For the reader's convenience, we include in this manuscript a number of standard facts about harmonic functions.

\section{Conformal questions and coverings}

A central result in Riemann surface theory is the uniformization theorem, which reduces the list of simply-connected Riemann surfaces to the Riemann sphere $\mathbb{S}^{2}$, the complex plane $\mathbb{C}$ and the upper halfplane $\mathbb{H}=\{(x, y) \in$ $\left.\mathbb{R}^{2} \mid y>0\right\}$. Since these model spaces have complete constant curvature metrics which are invariant under any group of conformal maps that acts properly discontinuously, it follows that every Riemann surface has a conformally related complete metric of constant curvature $1,-1$ or 0 . The so-called type problem consists of classifying Riemann surfaces which are not simply-connected. Assume the surface in question arises as the underlying Riemann surface of a complete embedded minimal surface $M \subset \mathbb{R}^{3}$ (so the universal covering $\widehat{M}$ of $M$ cannot be $\mathbb{S}^{2}$ ). Recently, Meeks and Rosenberg [19] proved that the case $\widehat{M}=\mathbb{C}$ can only occur when $M$ is the plane, catenoid or helicoid. Thus, from now on, we will assume that the universal covering of $M$ is $\mathbb{H}$.

A useful approach to study the type problem for such an $M$ once one is given a group $G$ of proper discontinuous isometries of $M$, is to consider 
relations between Liouville-type results on $M$ and on $M / G$ and to deduce conformal properties of $M$ from the behavior of the covering $M \rightarrow M / G$ and the quotient surface $M / G$. Of course, $M / G$ could be recurrent and $M$ being transient or admitting nonconstant positive and/or bounded harmonic functions. As a first example of the kind of results we are interested in, we state the following particular case of a theorem of Lyons ad Sullivan [8].

Proposition 2.1. Every properly immersed triply periodic minimal surface $(k=3)$ is transient and does not admit nonconstant positive harmonic functions.

Proof. Let $M$ be a properly immersed triply periodic minimal surface in $\mathbb{R}^{3}$, and $G$ be the group generated by three independent translations leaving $M$ invariant. Then, the corresponding covering $\pi: M \rightarrow M / G$ is abelian and $M / G$ is compact. By Theorem 1 in [8], $M$ does not admit nonconstant positive harmonic functions and is transient $\left(M / G\right.$ is compact and $G=\mathbb{Z}^{3}$ is a nilpotent group). This proves the proposition.

\section{Bounded harmonic functions}

We now study the existence of nonconstant bounded harmonic functions on properly embedded minimal surfaces in $\mathbb{R}^{3}$.

Proof of Theorems 1.1 and 1.2. Let $M \subset \mathbb{R}^{3}$ be a properly immersed minimal surface and let $G$ be a subgroup of orientation preserving isometries of $\mathbb{R}^{3}$ of rank 1 or 2 acting discontinuously on $M$. The group $G$ is either cyclic with generator a screw motion symmetry (possibly a translation) or $G$ is generated by two independent translations. In any case, the corresponding covering $M \rightarrow M / G$ is abelian. By Theorem 2 in [8], to obtain nonexistence of nonconstant bounded harmonic functions on $M$, it suffices to check that $M / G$ is recurrent.

First assume that $M$ is doubly periodic, so $G$ is generated by two independent translations. We do not lose generality by assuming these translations are horizontal. Then, the third coordinate function on $M$ descends to $M / G$ and defines a proper harmonic function. Since $(M / G) \cap x_{3}^{-1}[0, \infty)$ admits a proper positive harmonic function, it is a parabolic surface with boundary $^{2}$, and similarly for $(M / G) \cap x_{3}^{-1}(-\infty, 0]$. As $(M / G) \cap x_{3}^{-1}(0)$ is compact, we deduce that $M / G$ is recurrent.

\footnotetext{
${ }^{2}$ A Riemann surface $\Sigma$ with boundary is parabolic if its boundary has full harmonic measure, or equivalently, if bounded harmonic functions on $\Sigma$ are determined by their boundary values.
} 
Now assume $M$ is embedded, invariant by a translation or screw motion $T$ of $\mathbb{R}^{3}$ and has bounded curvature. Then, $M$ has an open regular neighborhood of positive radius (see [15] or [24]). Since the volume growth of $\mathbb{R}^{3} / T$ is quadratic and $M$ has bounded second fundamental form, the area growth of $M / G$ is at most quadratic. This condition implies $M / G$ is recurrent (see, e.g., Grigor'yan [6]). This finishes the proof.

\section{Recurrence and transience}

Our next goal is to prove Theorem 1.3. To do this, we shall need a result due to Epstein [4], which describes when a covering of a finitely punctured compact Riemann surface is either transient or recurrent, in terms of the group of deck transformations of the covering. Every Riemann surface $\Sigma$ of genus $g$ with $n+1>0$ punctures has the upper halfplane $\mathbb{H}=\{z=(x, y) \mid y>0\}$ as its universal covering (except in the cases $g=0$ with $n=0,1$ ). The first homology group $H_{1}(\Sigma)$ is given by $H_{\mathrm{c}} \times H_{\mathrm{e}}$, where $H_{\mathrm{c}}=\mathbb{Z}^{2 g}$ is the first homology group of the compactification of $\Sigma$ after attaching its ends and $H_{\mathrm{e}}=\mathbb{Z}^{n}$ is generated by small loops around all the ends of $\Sigma$ except one. Now suppose $\widetilde{\Sigma} \rightarrow \Sigma$ is a conformal covering with deck transformation group $G=\mathbb{Z}^{k}$. Since $G$ is abelian, $G$ can be viewed as a subgroup of $H_{1}(\Sigma)$. Let $p$ be the rank of the projection of $G$ over the factor $H_{\mathrm{e}}$. In this setting, Epstein [4] proved the following result.

$$
\widetilde{\Sigma} \text { is transient for Brownian motion if and only if } k+p \geq 3 \text {. }
$$

Proof of Theorem 1.3. Suppose $M \subset \mathbb{R}^{3}$ is a nonflat, $k$-periodic, properly embedded minimal surface such that $M / G \subset \mathbb{R}^{3} / G$ has finite topology. If $k=3$, then $M$ is transient by Proposition 2.1. If $k=0$, then $M$ is recurrent by the main result in [14], since it has finite genus. Now assume $k=1$. By Theorem 1 in [18], $M / G$ is conformally a finitely punctured compact Riemann surface. Let $g \geq 0$ and $n+1 \geq 2$ be, respectively, the genus of the compactification of $M / G$ and the number of ends, respectively. We can assume the universal covering of $M / G$ is $\mathbb{H}$ (otherwise $M$ is simply connected, which implies it is a plane or helicoid, both of which are conformally $\mathbb{C})$. Since the transformation group of the covering $M \rightarrow M / G$ is $\mathbb{Z}$, we have $p \leq 1$. Using (4.1), we deduce that $M$ is recurrent.

Now suppose $k=2$. By the description of the asymptotic geometry of any doubly periodic properly embedded minimal surface with finite topology quotient in Meeks and Rosenberg [17], the ends of $M / G$ divide into two families of parallel ends, all asymptotic to flat annuli (Scherk-type ends). 
Since the transformation group of the covering $M \rightarrow M / G$ is $\mathbb{Z} \oplus \mathbb{Z}$, we have $k=2$ and $p \leq 2$. When all the ends of $M$ are parallel, then $p=1$. Otherwise, $p=2$. Hence, $k+p \geq 3$ and $M$ is again transient by (4.1). Now Theorem 1.3 is proved.

Remark 4.1. In the proof of Theorems 1.1 and 1.2, we used Theorem 2 in [8], to reduce the nonexistence of nonconstant bounded harmonic functions on $M$, to the fact that $M / G$ is recurrent. In [8], Lyons and Sullivan give a similar result (Theorem 1) in order to deduce the nonexistence of nonconstant positive harmonic functions on $M$, which needs compactness of $M / G$. The hypotheses in their theorem cannot be weakened to recurrence, since there exist $\mathbb{Z}$-covers $\pi: \widetilde{M} \rightarrow M$, where $M$ is a recurrent Riemann surface and $\widetilde{M}$ does admit nonconstant positive harmonic functions. There is a simple example of these covers which is specially appropriate for this article since it is based on a complete embedded minimal surface in space. Consider a doubly periodic Scherk minimal surface $M \subset \mathbb{R}^{3}$. By Theorem 1.3, $M$ is transient for Brownian motion. Let $\mathbf{p}: M \rightarrow M / T$ be the $\mathbb{Z}$-covering obtained modulo one of the translations $T$ that leave $M$ invariant, and let $x$ be a point in $M / T$. Since $M / T$ has bounded curvature and $\mathbb{R}^{3} / T$ has quadratic volume growth, we deduce that $M / T$ has quadratic area growth. Hence, $M / T$ is recurrent and the same holds for $\Sigma=(M / T)-\{x\}$. Then, the restricted covering $\widetilde{\Sigma}=M-\mathbf{p}^{-1}(x) \rightarrow \Sigma$ has transformation group $\mathbb{Z}$, and $\widetilde{\Sigma}$ admits as a positive harmonic function the restriction of the Green function of $M$ with pole at an arbitrary point of $\mathbf{p}^{-1}(x)$.

\section{Positive harmonic functions}

Our main goal in this section is to prove Theorem 1.4, for which we will use some well-known results about positive harmonic functions (see, for instance, [9] and [10]). The first step in the study of the existence of nonconstant positive harmonic functions on a Riemann surface is to reduce to a particular case of such functions.

Definition 5.1. Let $M$ be a Riemann surface. A harmonic function $h$ : $M \rightarrow \mathbb{R}^{+}$is called minimal if the only harmonic functions $u: M \rightarrow \mathbb{R}^{+}$below any constant multiple of $h$ are multiples of $h$.

Proposition 5.2. If a Riemann surface $M$ admits a positive nonconstant harmonic function, then it admits a minimal nonconstant positive harmonic function. 
Proof. Fix a point $p \in M$ and consider the set $\mathcal{H}$ of all positive harmonic functions having the value 1 at $p$. Clearly $\mathcal{H}$ is nonvoid $(1 \in \mathcal{H})$ and convex. A direct application of the Harnack inequality gives that $\mathcal{H}$ is compact in the uniform topology on compact subsets of $M$. By the Krein-Milman theorem, $H$ equals the convex hull of the set of its extremal elements (recall that an element $h \in \mathcal{H}$ is called extremal if whenever $h=\theta h_{1}+(1-\theta) h_{2}$ for some $\theta \in[0,1]$ and $h_{1}, h_{2} \in H$, then $\theta=0$ or $\left.\theta=1\right)$. By assumption, $\mathcal{H}$ does not reduce to $\{1\}$, and hence there exists an extremal element $h \in \mathcal{H}$, with $h \neq 1$ (in particular, $h$ is not constant). It remains to prove that $h$ is a minimal harmonic function. To see this, let $u$ be a positive harmonic function with $u \leq c h$ for some $c>0$. After exchanging the constant $c$, we can assume $u \in \mathcal{H}$, and so the minimality of $h$ reduces to checking that $u=h$. Since $c h-u$ is harmonic and nonnegative, the maximum principle implies that either $u=c h$ (hence $c=1$ and $u=h$ ) or $c h-u>0$ in $M$. Arguing by contradiction, assume the last inequality holds. Evaluating at $p$, we have $c>1$. Now consider the functions $u,(c h-u) /(c-1)$, both in $\mathcal{H}$. Since $1 / c \in(0,1)$ and

$$
\frac{1}{c} u+\left(1-\frac{1}{c}\right) \frac{c h-u}{c-1}=h
$$

we have written $h$ as a nontrivial convex linear combination of $u$, $(c h-u) /(c-1)$, which contradicts the extremality of $h$.

Next, we give the outline of the proof of Theorem 1.4. Reasoning by contradiction, we assume that our properly embedded minimal surface $M \subset \mathbb{R}^{3}$ admits a nonconstant positive harmonic function and, therefore, a minimal nonconstant positive harmonic function $h$. Recall that we are assuming that $M / G$ has finite topology. Note that $G$ cannot reduce to the identity map (otherwise $M$ has finite topology and so, it is recurrent by Theorem 1 in [14]), and neither can it have rank 1 (in such a case, $M$ is again recurrent, now by Theorem 1.3) nor have rank 3 (by Proposition 2.1). Hence, we can assume $M$ is doubly periodic.

If the harmonic function $h$ descended to $M / G$, we would obtain a contradiction, since $M / G$ has finite total curvature [17], and so, it is recurrent. The condition for $h$ to descend is that $h \circ \phi=h$ for every isometry $\phi \in G$. Instead of proving this equality, we will show a weaker condition, namely

$$
h \circ \phi=c h \quad \text { in } M
$$

where $c$ is a positive number depending on $\phi \in G$. This equality will imply that the sum of $\log h$ with a certain coordinate function of $M$ descends to 
$M / G$, and we will show that such condition implies $h$ is constant, which is the desired contradiction. The proof of Equation (5.1) follows by applying the minimality of $h$, provided one has proved that for any $\phi \in G$, there exists $c_{1}=c_{1}(\phi)>0$ such that

$$
h \circ \phi \leq c_{1} h \text { in } M \text {. }
$$

This inequality (5.2) will be proved in two steps: first on a compact set $W \subset M$ such that the closure of any component $E$ of $M-W$ is conformally a halfspace. Secondly, we will work on any such component $E$, propagating the inequality (5.2) from $\partial E$ to the whole end $E$. Next, we enter into the details of what we have briefly explained.

Lemma 5.3. Let $\phi: \Sigma \rightarrow \Sigma$ be a holomorphic map on a Riemann surface $\Sigma$ that admits a hyperbolic metric. Suppose that there exists $c=c(\phi)>0$ such that $d_{-1}(\phi(x), x) \leq c$ for all $x \in \Sigma$ (here $d_{-1}$ denotes hyperbolic distance). Then, every positive minimal harmonic function $h$ on $\Sigma$ satisfies that $h \circ \phi$ is a multiple of $h$.

Proof. Given $x \in \Sigma$, fix a compact set $K \subset \Sigma$ such that $x, \phi(x) \in K$. By the Harnack inequality, we have

$$
h(\phi(x)) \leq \sup _{K} h \leq c_{1} \inf _{K} h \leq c_{1} h(x),
$$

where $c_{1}>0$ depends only on $d_{-1}(\phi(x), x)$. Since $d_{-1}(\phi(x), x)$ is bounded for $x \in \Sigma$, we obtain a number $c_{2}>0$ such that $h \circ \phi \leq c_{2} h$. Now the lemma follows from the fact that $h \circ \phi$ is positive harmonic and $h$ is minimal.

The main hypothesis in Lemma 5.3 is to have a control on the hyperbolic distance in $\Sigma$. When $\Sigma$ is the underlying Riemann surface of a complete minimal surface of bounded Gaussian curvature in a complete flat threemanifold, then it will be enough to bound the intrinsic distance on the surface, as stated in the next result due to Yau [25].

Lemma 5.4. Let $d s^{2}$ be a complete metric on a surface $\Sigma$, being conformal to the hyperbolic metric $d s_{-1}^{2}$. Assume the Gauss curvature of $d s^{2}$ satisfies $K \geq-c$ for some $c>0$. Then,

$$
d s_{-1}^{2} \leq c d s^{2}
$$

Lemma 5.5 (Representation formula). Let $\Sigma$ be a parabolic Riemann surface with boundary and let $u \geq 0$ be a continuous function on $\Sigma$, harmonic 
in $\Sigma-\partial \Sigma$. Let $\mu_{p}, p \in \Sigma-\partial \Sigma$, be the harmonic measure on $\partial \Sigma$. Then, there exists a continuous function $v: \Sigma \rightarrow[0, \infty)$, harmonic on $\Sigma-\partial \Sigma$ such that $v=0$ on $\partial \Sigma$ and

$$
u(p)=\int_{\partial \Sigma} u d \mu_{p}+v(p) \quad \forall p \in \Sigma-\partial \Sigma .
$$

Proof. Let $\varphi: \Sigma \rightarrow[0,1]$ be a smooth cut-off function (i.e., $\varphi=1$ on a compact set $\Omega$ and has compact support) and let $u_{\varphi}$ be the bounded continuous function on $\Sigma$, harmonic on $\Sigma-\partial \Sigma$, given by

$$
u_{\varphi}(p)=\int_{\partial \Sigma} \varphi u d \mu_{p}
$$

Take an exhaustion of $\Sigma$ by smooth relatively compact domains $\Omega_{k} \nearrow \Sigma$. Let $u_{k}$ be the solution of the Dirichlet problem

$$
\begin{cases}\Delta u_{k}=0 & \text { in } \Omega_{k} \\ u_{k}=\varphi u & \text { in } \partial \Omega_{k} \cap \partial \Sigma \\ u_{k}=0 & \text { in } \partial \Omega_{k}-\partial \Sigma .\end{cases}
$$

Then, $u_{k} \leq u_{\varphi}$ (which is bounded). Since $u \geq 0$, then $\left\{u_{k}\right\}_{k}$ is monotonically nondecreasing. Hence, the $\operatorname{limit}_{\lim _{k}} u_{k}$ exists and is a bounded harmonic function. Since $\Sigma$ is parabolic, then $\lim _{k} u_{k}$ is determined by its boundary values, which coincide with those of $u_{\varphi}$. As $u_{\varphi}$ is also bounded, we have $\lim _{k} u_{k}=u_{\varphi}$. As $u_{k} \leq u$, taking limits, we have $u_{\varphi} \leq u$, i.e.,

$$
\int_{\partial \Sigma} \varphi u d \mu_{p} \leq u(p), \text { for all } p \in \Sigma-\partial \Sigma
$$

Now let $\varphi \rightarrow 1$, and note that Fatou's lemma implies $u$ is $\mu_{p}$-integrable in $\partial \Sigma$ and

$$
u_{1}(p):=\int_{\partial \Sigma} u d \mu_{p}
$$

is a harmonic function that coincides with $u$ on $\partial \Sigma$, and $u_{1} \leq u$. Now our lemma follows by taking $v=u-u_{1}$.

Lemma 5.6. In the situation of Lemma 5.5, assume that $\Sigma$ is conformally $\{(x, y) \mid y \geq 0\}$. Then, the function $v$ in the representation formula is a multiple of $y$. 
Proof. Let $v: \Sigma \rightarrow[0, \infty)$ be a continuous function, harmonic on $\{y>0\}$ and vanishing at $\{y=0\}$. By the boundary maximum principle, the positivity of $v$ implies $\partial v / \partial y>0$ along the boundary. Let $v^{*}$ be its harmonic conjugate function. So $f=v+i v^{*}$ is holomorphic and can be extended by Schwarz reflection to the entire complex plane. Furthermore, $f$ maps monotonically the real axis into the the imaginary axis, and no points of $\mathbb{C}-\mathbb{R}$ can be mapped by $f$ into $i \mathbb{R}$. Therefore, $f$ is linear and $v$ is linear as well.

Question 5.7. Is Lemma 5.6 true for the intersection of a properly immersed minimal surface in $\mathbb{R}^{3}$ with a closed halfspace and the harmonic function $v$ in the representation formula being a multiple of the height function over its boundary? By Theorem 3.1 in [2], such an intersection is parabolic.

Proof of Theorem 1.4. The only case that remains open is when $M \subset \mathbb{R}^{3}$ is a properly embedded doubly periodic minimal surface, invariant by the group $G$ generated by two independent translations, which we can assume horizontal. Suppose $M$ admits a nonconstant positive harmonic function $h$. By Proposition 5.2, we can assume $h$ is minimal.

Claim. For every $\phi \in G$, there exists $c=c(\phi)>0$ such that $h \circ \phi=c h$ in $M$.

Proof of the claim. Fix $\phi \in G$. Since $h$ is positive, minimal and harmonic, the claim follows if we prove that $h \circ \phi \leq c_{1} h$ in $M$, for some $c_{1}>0$.

Consider a closed horizontal slab $W \subset \mathbb{R}^{3}$ of finite width. Since $M \cap$ $W$ is invariant by $\phi$ and $W / G$ is compact, the function $x \in M \cap W \mapsto$ $d_{M}(\phi(x), x)$ is bounded (here $d_{M}$ denotes intrinsic distance on $M$ ). Since $M / G$ has finite topology, it has finite total curvature, and so, it has bounded curvature. Since $M$ also has bounded curvature, Lemma 5.4 implies that the hyperbolic metric on $M$ is bounded from above by a multiple of the induced metric on $M$ (i.e., the restriction to $M$ of the usual inner product in $\left.\mathbb{R}^{3}\right)$. Thus, the function $x \in M \cap W \mapsto d_{-1}(\phi(x), x)$ is bounded, where $d_{-1}$ denotes hyperbolic distance. In this situation, the proof of Lemma 5.3 gives that

$$
(h \circ \phi)(x) \leq c_{1} h(x) \text { for all } x \in M \cap W
$$

for some $c_{1}>0$.

We next show that we can propagate the inequality (5.3) to the ends of $M$. Let $E$ be the representative of an end of $M$, obtained after intersection of $M$ with the closed upper halfspace $\left\{x_{3} \geq a\right\}$ such that $\left\{x_{3}=a\right\} \subset \partial W$ (for the lower halfspace in the complement of $W$, the argument is the same). 
Note that $E$ is parabolic and conformally a halfspace. Applying Lemmas 5.5 and 5.6, we find $c_{2} \geq 0$ such that

$$
h(p)=\int_{\partial E} h d \mu_{p}+c_{2}\left(x_{3}(p)-a\right), \quad \text { for all } p \in E-\partial E,
$$

where $d \mu_{p}$ stands for the harmonic measure on $\partial E$ associated to $p \in E-\partial E$.

If $c_{2}>0$, then consider the nonnegative subharmonic function $H: M \rightarrow$ $[0, \infty]$ equal to $c_{2}\left(x_{3}(p)-a\right)$ on $E$ and 0 elsewhere on $M$. Note that $H \leq h$ on $M$ and $H$ is invariant under the translation $\tau \in G$ which leaves $E$ invariant. Consider the collection $\mathcal{H}$ of all nonnegative subharmonic functions on $M$ that lie below $h$ and which are invariant under $\tau$. Next, we check that the Perron method can be applied to $\mathcal{H}$. Take $h_{1} \in \mathcal{H}$ and a small disk $D$ in the interior of a fundamental domain of $\tau$ in $M$. Let $D_{\tau}$ be the $\tau$-orbit of $D$. Let $h_{2}$ be the harmonic function on $D_{\tau}$ with the same boundary values as $\left.h_{1}\right|_{\partial D_{\tau}}$. Note that $h_{2} \geq h_{1}$ on the interior of $D_{\tau}$ and $h_{2}$ is $\tau$-invariant. By the maximum principle, $h_{2}$ lies below $\left.h\right|_{D_{\tau}}$ since the boundary values of $h_{2}$ lie below those of $h$ and each component of $D_{\tau}$ is compact. Therefore, the function $h_{3}$ on $M$, which is equal to $h_{1}$ on $M-D_{\tau}$ and equal to $h_{2}$ on $D_{\tau}$, lies in $\mathcal{H}$, and so, the Perron method is applicable to $\mathcal{H}$. Thus, the supremum of $\mathcal{H}$ is a positive harmonic function $v$ on $M$ such that $v \circ \tau=v$ and $H<v \leq h$. Since $v$ descends to a nonconstant positive harmonic function on $M /\langle\tau\rangle \subset \mathbb{R}^{3} /\langle\tau\rangle$, we contradict that $M /\langle\tau\rangle$ is recurrent by Theorem 1.1. Thus, we may assume that $c_{2}=0$. This means that

$$
h(p)=\int_{\partial E} h d \mu_{p}, \quad \text { for all } p \in E-\partial E .
$$

For $p \in E-\partial E$, Equation (5.5) gives

$$
\begin{aligned}
h(\phi(p)) & =\int_{\partial E} h d \mu_{\phi(p)}=\int_{\phi(\partial E)} h d \mu_{\phi(p)}=\int_{\partial E}(h \circ \phi) \phi^{*} d \mu_{\phi(p)} \\
& =\int_{\partial E}(h \circ \phi) d \mu_{p} \stackrel{(5.3)}{\leq} c_{1} \int_{\partial E} h d \mu_{p} \stackrel{(5.5)}{=} c_{1} h(p),
\end{aligned}
$$

and the claim is proved. Now Theorem 1.4 follows directly from the above claim and Proposition 5.8.

Proposition 5.8. Let $M \subset \mathbb{R}^{3}$ be a properly immersed doubly periodic minimal surface and $h: M \rightarrow \mathbb{R}^{+}$a positive smooth superharmonic function. If for any translation $\phi$ that leaves $M$ invariant, there exists $c(\phi)>0$ such that $h \circ \phi=c(\phi) h$ in $M$, then $h$ is constant. 
Proof. Take two independent translations $\phi_{1}, \phi_{2}$ that leave $M$ invariant. By hypothesis, $\log \left(h \circ \phi_{i}\right)=\log h+\log c_{i}$ for certain $c_{i}>0, i=1,2$. Since the periods of $M$ in the directions of $\phi_{1}, \phi_{2}$ are independent, elementary linear algebra gives a linear combination $x$ of the coordinate functions $x_{1}, x_{2}$ associated to such period vectors, such that the periods of $x$ in $\phi_{1}, \phi_{2}$ coincide with those of $\log h$. Then the function $v=\log h-x$ descends to the quotient of $M$ by the group $G$ generated by $\phi_{1}, \phi_{2}$. Since $h=e^{v+x}$ is superharmonic on $M$, we have

$$
\Delta v+|\nabla v+\nabla x|^{2} \leq 0 \quad \text { in } M / G
$$

Take a smooth nonnegative compactly supported function $\varphi$ on $M / G$. Then

$$
\begin{aligned}
\int_{M / G} \varphi^{2}|\nabla v+\nabla x|^{2} & \leq-\int_{M / G} \varphi^{2} \Delta v=-\int_{M / G} \varphi^{2} \Delta(v+x) \\
& =2 \int_{M / G} \varphi\langle\nabla \varphi, \nabla v+\nabla x\rangle \leq 2 \int_{M / G} \varphi|\nabla \varphi||\nabla v+\nabla x| \\
& \leq 2\left(\int_{M / G} \varphi^{2}|\nabla v+\nabla x|^{2}\right)^{1 / 2}\left(\int_{M / G}|\nabla \varphi|^{2}\right)^{1 / 2} .
\end{aligned}
$$

After simplifying, we have

$$
\int_{M / G} \varphi^{2}|\nabla v+\nabla x|^{2} \leq 4 \int_{M / G}|\nabla \varphi|^{2}
$$

Given $0<t<s$, take the cut-off function $\varphi$ on $M / G$ so that

$$
\varphi= \begin{cases}1 & \text { in }\left\{\left|x_{3}\right| \leq t\right\} \\ \frac{s-\left|x_{3}\right|}{s-t} & \text { in }\left\{t \leq\left|x_{3}\right| \leq s\right\} \\ 0 & \text { in }\left\{s \leq\left|x_{3}\right|\right\}\end{cases}
$$

Then (5.6) gives

$$
\begin{aligned}
\int_{\left\{\left|x_{3}\right| \leq t\right\}}|\nabla v+\nabla x|^{2} & \leq \frac{4}{(s-t)^{2}} \int_{\left\{t \leq\left|x_{3}\right| \leq s\right\}}\left|\nabla x_{3}\right|^{2} \\
& =\frac{4}{(s-t)^{2}} \int_{\left\{t \leq\left|x_{3}\right| \leq s\right\}} \operatorname{div}\left(x_{3} \nabla x_{3}\right)
\end{aligned}
$$




$$
\begin{aligned}
& =\frac{4}{(s-t)^{2}}\left(s \int_{\left\{\left|x_{3}\right|=s\right\}} \frac{\partial x_{3}}{\partial \eta}-t \int_{\left\{\left|x_{3}\right|=t\right\}} \frac{\partial x_{3}}{\partial \eta}\right) \\
& =\frac{4}{(s-t)^{2}}(s+t) \operatorname{Flux}\left(x_{3}\right),
\end{aligned}
$$

where $\operatorname{Flux}\left(x_{3}\right)$ is the scalar flux of $x_{3}$ along $(M / G) \cap\left\{\left|x_{3}\right|=s\right\}$ (which does not depend on $s$ by the divergence theorem). Taking $s \rightarrow \infty$, we conclude $\nabla v+\nabla x=0$ in $(M / G) \cap\left\{\left|x_{3}\right| \leq t\right\}$ (and then in all of $M / G$ after taking $t \rightarrow \infty)$. Thus, $v+x$ is constant in $M / G$ and the same holds for $h$ in $M$.

\section{6. a-Stable minimal surfaces}

We first recall a well-known characterization of $a$-stable minimal surfaces, essentially due to Fischer-Colbrie and Schoen [5] (in fact, the following statement generalizes directly to operators $\Delta+q$, where $q$ is a smooth function on $M)$.

Lemma 6.1. Let $M$ be a complete minimal surface in a complete flat threemanifold $N$. Then, the following conditions are equivalent:

1. $M$ is a-stable.

2. There exists a positive solution $u$ of $\Delta u-a K u=0$ on $M$.

3. There exists a positive solution $u$ of $\Delta u-a K u \leq 0$ on $M$.

Proof. The equivalence between 1 and 2 is stated in Theorem 1 in [5], and clearly 2 implies 3 . It then remains to prove that 3 implies 1 . Let $v$ be a compactly supported smooth function on $M$. Since $u>0$ on $M$, the function $\varphi=v / u$ is smooth with compact support, and integration by parts give

$$
\begin{aligned}
\int_{M}\left(|\nabla v|^{2}+a K v^{2}\right) & =\int_{M}\left(|\nabla(\varphi u)|^{2}+a K \varphi^{2} u^{2}\right) \\
& =\int_{M}\left(-\varphi u \Delta(\varphi u)+a K \varphi^{2} u^{2}\right) \\
& =\int_{M}\left(-\varphi^{2} u \Delta u-2\langle\nabla \varphi, \nabla u\rangle \varphi u-u^{2} \varphi \Delta \varphi+a K \varphi^{2} u^{2}\right) \\
& \geq-\int_{M}\left(\frac{1}{2}\left\langle\nabla\left(\varphi^{2}\right), \nabla\left(u^{2}\right)\right\rangle+u^{2} \varphi \Delta \varphi\right) \\
& =\int_{M}|\nabla \varphi|^{2} u^{2} \geq 0,
\end{aligned}
$$

thus $M$ is $a$-stable. 
Proof of Proposition 1.5. Let $M$ be an $a$-stable minimal surface in a complete flat three-manifold $N$. By Lemma 6.1, there exists a smooth positive function $u$ on $M$ such that $\Delta u=a K u$. Since $N$ is flat, then $K$ is nonpositive and so, $u$ is superharmonic. Since we are assuming that $M$ is not flat, then $K$ is negative at some point, and so, $u$ cannot be constant. Since the existence of such a nonconstant positive superharmonic function on $M$ is equivalent to the property that $M$ is transient for Brownian motion, our proposition is proved.

Proof of Theorem 1.6. Suppose $M$ is a complete, non totally geodesic, $a$ stable minimal surface with finite genus in a complete flat three-manifold $N$.

If $M$ does not have bounded curvature, then the Local Picture Theorem on the Scale of Curvature (Theorem 1.3 in [12]) produces a sequence of rescaled compact subdomains of $M$ obtained as components of the intersection of $M$ with appropriately chosen small extrinsic balls of $N$, and that sequence converges $C^{k}$ on compact subsets of $\mathbb{R}^{3}$ with multiplicity one to a connected properly embedded minimal surface $M_{\infty} \subset \mathbb{R}^{3}$ with $\overrightarrow{0} \in M_{\infty}$, $\left|K_{M_{\infty}}\right| \leq 1$ on $M_{\infty}$ and $\left|K_{M_{\infty}}\right|(\overrightarrow{0})=1$ (here $\left|K_{M_{\infty}}\right|$ denotes the absolute Gaussian curvature of $M_{\infty}$ ). So, after possibly replacing $M$ by such a local picture dilation limit on the Scale of Curvature, we can assume that $M$ has bounded curvature (the $a$-stability property is preserved under smooth dilation limits). By Proposition 1.5, to obtain a contradiction, we just need to prove that $M$ is recurrent for Brownian motion.

Since $M$ is a complete embedded minimal surface of bounded curvature in a flat three-manifold $N$ and $M$ is not totally geodesic, then $M$ is proper (the closure of $M$ is a minimal lamination $\mathcal{L}$ of bounded curvature of $N$; if $M$ were not proper, then $\mathcal{L}$ would have a limit leaf $L$ and both $\mathcal{L}$ and $L$ would lift to a similar nonflat minimal lamination of $\mathbb{R}^{3}$ with bounded curvature and a limit leaf, which contradicts Theorem 1.6 in [19]).

If $N$ is $\mathbb{R}^{3}$, then $M$ is recurrent for Brownian motion because it is properly embedded with finite genus (see Theorem 1 in [14]). Assume now that $M$ has finite genus and bounded curvature and $N$ is not $\mathbb{R}^{3}$. After lifting to a finite cover, we may assume that $N$ is $\mathbb{R}^{3} / S_{\theta}, \mathbb{R}^{2} \times \mathbb{S}^{1}$ or $\mathbb{T}^{2} \times \mathbb{R}$, where $S_{\theta}$ is a screw motion symmetry of infinite order and $\mathbb{T}^{2}$ is a flat twotorus. Since a properly embedded minimal surface of bounded curvature in a complete flat three-manifold has a fixed size embedded regular neighborhood whose intrinsic volume growth is comparable to the intrinsic area growth of the surface (i.e., the ratio of both growths is bounded above and below by positive constants), then the intrinsic area growth of $M$ is at most quadratic since the volume growth of $\mathbb{R}^{3} / S_{\theta}, \mathbb{R}^{2} \times \mathbb{S}^{1}$ and of $\mathbb{T}^{2} \times \mathbb{R}$ is at most quadratic; see the proof of Theorems 1.1 and 1.2 in Section 3. Since $M$ 
has at most quadratic area growth, it is recurrent for Brownian motion [6]. This completes the proof of Theorem 1.6.

Following the lines in former sections, we are interested in natural relations between covering maps and the notion of $a$-stability. Some of these relations are contained in the following result.

Lemma 6.2 (a-stability lemma). Let $M \subset N^{3}$ be a complete minimal surface in a complete flat three-manifold.

1. If $M$ is a-stable, then any covering space of $M$ is also a-stable.

2. If $M$ is a-unstable and $\widetilde{M}$ is a covering space of $M$ such that the components of the inverse image of each compact subdomain of $M$ have subexponential area growth, then $\widetilde{M}$ is also a-unstable (for example, if $\widetilde{M}$ is a finitely generated abelian cover, then it satisfies this subexponential area growth property).

Proof. As $a$-stability is characterized by the existence of a positive solution on $M$ of $\Delta u-a K u=0$, then item (1) follows directly by lifting $u$ to $\widetilde{M}$.

We now consider statement (2). First note that since $M$ is $a$-unstable, there exists a smooth compact subdomain $\Omega \subset M$ such that the first eigenvalue $\lambda_{1}$ of the $a$-stability operator $\Delta-a K$ is negative. Denote by $v$ the first eigenfunction of the $a$-stability operator for $\Omega$ with zero boundary values. Therefore, $\Delta v-a K v+\lambda_{1} v=0$, with $\lambda_{1}<0$.

Let $\widetilde{\Omega} \subset \widetilde{M}$ be the pullback image of $\Omega$ by the covering map $\pi: \widetilde{M} \rightarrow M$ and let $u=v \circ \pi$ be the lifted function of $v$ on $\widetilde{\Omega}$. Thus,

$$
\Delta u-a K u+\lambda_{1} u=0 \quad \text { in } \widetilde{\Omega}, \quad \text { and } u=0 \quad \text { in } \partial \widetilde{\Omega} .
$$

Let $\varphi$ be a compactly supported smooth function on $\widetilde{M}$. Arguing as in the proof of Lemma 6.1, Equation (6.1) implies

$$
\int_{\widetilde{\Omega}}\left(|\nabla(\varphi u)|^{2}+a K \varphi^{2} u^{2}\right)=\int_{\widetilde{\Omega}}\left(\lambda_{1} \varphi^{2} u^{2}+|\nabla \varphi|^{2} u^{2}\right) .
$$

Reasoning by contradiction, assume that $\widetilde{M}$ is $a$-stable. Then, the last integral is nonnegative, and we conclude that

$$
-\lambda_{1} \int_{\widetilde{\Omega}} \varphi^{2} u^{2} \leq \int_{\widetilde{\Omega}}|\nabla \varphi|^{2} u^{2}
$$

Denote by $r: \widetilde{M} \rightarrow[0, \infty)$ the Riemannian distance to a fixed point $q \in \widetilde{M}$ and $B(R)=\{r \leq R\}$ the corresponding intrinsic geodesic ball. Consider the 
cut-off Lipschitz function $\varphi_{R}$, defined by

$$
\varphi_{R}= \begin{cases}1 & \text { in } B(R), \\ 0 & \text { in } \widetilde{M}-B(R+1), \\ R+1-r & \text { in } B(R+1)-B(R) .\end{cases}
$$

By a standard density argument, we can take $\varphi=\varphi_{R}$ in (6.2) and obtain, for almost any $R>0$,

$$
-\lambda_{1} \int_{\widetilde{\Omega} \cap B(R)} u^{2} \leq \int_{\widetilde{\Omega} \cap B(R+1)} u^{2}-\int_{\widetilde{\Omega} \cap B(R)} u^{2},
$$

which is impossible as the hypothesis implies that the function

$$
R \longmapsto \int_{\widetilde{\Omega} \cap B(R)} u^{2}
$$

has at least exponential growth. This fact together with the boundedness of $u$ and the subexponential growth of the area of $\widetilde{\Omega} \cap \mathrm{B}(\mathrm{R})$ lead to a contradiction. This proves the lemma.

Our next goal is to prove Theorem 1.8 stated in the introduction. To do this, we first recall some definitions and results from [12]. A dilation $d: \mathbb{R}^{3} \rightarrow \mathbb{R}^{3}$ is a conformal diffeomorphism that can be expressed uniquely by $d(x)=\lambda(x-p)$ for some $p \in \mathbb{R}^{3}$ and positive number $\lambda$. The space $D(M)$ of dilation limits of a properly embedded minimal surface $M \subset \mathbb{R}^{3}$ is the set of properly embedded nonflat minimal surfaces $\Sigma \subset \mathbb{R}^{3}$ which are smooth limits on compact sets in $\mathbb{R}^{3}$ of a divergent sequence of dilations of $M$ (a sequence of dilations $d_{n}(x)=\lambda_{n}\left(x-p_{n}\right)$ is divergent if $p_{n} \rightarrow \infty$ as $\left.n \rightarrow \infty\right)$. A surface $\Sigma \in D(M)$ is a minimal element if $D(\Sigma)$ is a minimal (smallest) $D$-invariant subset of $D(M)$ (a subset $\Delta \subset D(M)$ is $D$ invariant if $D\left(\Sigma^{\prime}\right) \subset$ $\Delta$ whenever $\Sigma^{\prime} \in \Delta$ ). The Dynamics Theorem for embedded minimal surfaces (Theorem 1.6 in [12]) states that if $M$ does not have finite total curvature and if no surface in $D(M)$ has finite total curvature, then there exists a minimal element $\Sigma \in D(M)$ which has bounded curvature and $\Sigma \in D(\Sigma)$. In particular, for such a $\Sigma$, there exists a divergent sequence of dilations $d_{n}$ such that the surfaces $\Sigma(n)=d_{n}(\Sigma)$ converge smoothly to $\Sigma$ on compact subsets of $\mathbb{R}^{3}$ and so, we call such a $\Sigma$ quasi-dilation-periodic.

Proof of Theorem 1.8. Let $M$ be a complete embedded nonflat $a$-stable minimal surface in a complete flat three-manifold. By Theorem 1.6, $M$ must have infinite genus. Since $a$-stability is preserved under homotheties, limits and taking finitely generated abelian covers (by part (2) of Lemma 6.2), the 
Local Picture Theorem on the Scale of Curvature implies that there exists a nonflat properly embedded $a$-stable minimal surface $M_{1} \subset \mathbb{R}^{3}$ of bounded curvature. By Theorem 1.6, such a surface $M_{1}$ must have infinite genus. In particular, $M_{1}$ cannot have finite total curvature. Now consider the set $D\left(M_{1}\right)$ of dilation limits of $M_{1}$. Since any surface in $D\left(M_{1}\right)$ is $a$-stable, the above argument shows that no surface in $D\left(M_{1}\right)$ has finite total curvature. By the Dynamics Theorem, there exists a minimal element $\Sigma \in D\left(M_{1}\right)$ with bounded Gaussian curvature, which is quasi-dilation-periodic. Since $\Sigma \in D\left(M_{1}\right)$, then $\Sigma$ is $a$-stable (so it has infinite genus by Theorem 1.6). To finish the proof, it only remains to check that no such $\Sigma$ is 1-periodic.

Reasoning by contradiction, assume $\Sigma$ is invariant by a translation or screw motion symmetry $T$. Since the volume growth of $\mathbb{R}^{3} / T$ is quadratic and the proper surface $\Sigma / T \subset \mathbb{R}^{3} / T$ admits a regular neighborhood of positive radius, then $\Sigma / T$ has at most quadratic area growth, and thus, it is recurrent for Brownian motion. In particular, $\Sigma / T$ is $a$-unstable. By part (2) of Lemma 6.2, we deduce that $\Sigma$ is also $a$-unstable, a contradiction. Now Theorem 1.8 is proved.

If we do not assume embeddedness, then there are nonflat complete $a$-stable surfaces in $\mathbb{R}^{3}$. The following lemma gives us a way to obtain some of these.

Lemma 6.3. If a complete orientable minimal surface $M$ in $\mathbb{R}^{3}$ is simply connected and its Gauss map omits three spherical values, then $M$ is a-stable for some $a>0$ depending only on the omitted values.

Proof. We can assume that $M$ is not flat, hence $M$ is conformally the unit disk (since the Gauss map of $M$ omits three values). So, we can consider on $M$ the complete hyperbolic metric $d s_{h}^{2}$ of constant curvature -1 . It is known that for any compactly supported smooth function $u$, we have $\int_{M}\left|\nabla_{h} u\right|^{2} d A_{h} \geq 1 / 4 \int_{M} u^{2} d A_{h}$, where the length $\left|\nabla_{h} u\right|$ of the gradient of $u$ and the measure $d A_{h}$ are taken with respect to the metric $d s_{h}^{2}$. On the other hand, as the Gauss map $N$ omits three values, we have that $\left|\nabla_{h} N\right| \leq c$ for some constant $c$ depending only on the omitted values, see [22]. Therefore, we obtain that $\int_{M}\left|\nabla_{h} u\right|^{2} d A_{h} \geq 1 /\left(4 c^{2}\right) \int_{M}\left|\nabla_{h} N\right|^{2} u^{2} d A_{h}$, which, due to the conformal invariance of the Dirichlet integral and using that $|\nabla N|^{2}=$ $-2 K$, implies that $M$ is $\left(4 c^{2}\right)^{-1}$-stable.

For instance, by Lemma 6.3 , the universal covering $\widetilde{M}$ of any doubly periodic Scherk minimal surface $M \subset \mathbb{R}^{3}$ is $a$-stable for some $a>0$. Nevertheless, $M$ itself is $a$-unstable for all values $a>0$. In fact, this property remains valid for any doubly periodic minimal surface, as shown in the next result. 
Proposition 6.4. Let $M \subset \mathbb{R}^{3}$ be a properly immersed doubly periodic nonflat minimal surface. Then, $M$ is a-unstable for any $a>0$.

Proof. Let $G$ be the rank 2 group of translations leaving $M$ invariant. Then, $M / G$ is a properly immersed minimal surface in $\mathbb{R}^{3} / G=\mathbb{T}^{2} \times \mathbb{R}$. Since the natural height function $h: \mathbb{T}^{2} \times \mathbb{R} \rightarrow \mathbb{R}$ restricts to a proper harmonic function on the ends of $M / G$, we deduce that $M / G$ is recurrent for Brownian motion. By Proposition 1.5, $M / G$ is $a$-unstable. Since the covering $M \rightarrow$ $M / G$ is finitely generated and abelian, part (2) of Lemma 6.2 implies that $M$ is also $a$-unstable.

Remark 6.5. Any complete embedded doubly periodic nonflat minimal surface $M \subset \mathbb{R}^{3}$ with finite topology in the quotient $\mathbb{T}^{2} \times \mathbb{R}$ is transient (by Theorem 1.3), which implies that there exists a nonconstant positive superharmonic function $u$ on $M$, although such a $u$ cannot satisfy $\Delta u-$ $a K u \leq 0$ by Lemma 6.1 and Proposition 6.4. Finally, although $M$ is not recurrent, it comes close to satisfying that condition, since positive harmonic functions on it are constant (by Theorem 1.4).

\section{Acknowledgments}

The second and third authors' research is partially supported by a MEC/FEDER grant no. MTM2004-02746.

\section{References}

[1] T. H. Colding and W. P. Minicozzi II, The Calabi-Yau conjectures for embedded surfaces, Preprint, math. DG/0404197.

[2] P. Collin, R. Kusner, W. H. Meeks III, and H. Rosenberg, The geometry, conformal structure and topology of minimal surfaces with infinite topology, J. Diff. Geom. 67 (2004), 377-393.

[3] M. do Carmo and C. K. Peng, Stable minimal surfaces in $\mathbb{R}^{3}$ are planes, Bull. AMS 1 (1979), 903-906, MR0546314, Zbl 442.53013.

[4] C. L. Epstein, Positive harmonic functions on abelian covers, J. Funct. Anal. 82(2), (1989), 303-315.

[5] D. Fischer-Colbrie and R. Schoen, The structure of complete stable minimal surfaces in 3-manifolds of non-negative scalar curvature, 
Comm. Pure Appl. Math. 33 (1980), 199-211, MR0562550, Zbl 439.53060 .

[6] A. Grigor'yan, Analytic and geometric background of recurrence and non-explosion of Brownian motion on Riemannian manifolds, Bull. AMS 36(2) (1999), 135-249.

[7] S. Kawai, Operator $\Delta-a K$ on surfaces, Hokkaido Math. J. 17(2) (1988), 147-150.

[8] T. Lyons and D. Sullivan, Function theory, random paths, and covering spaces, J. Diff. Geom. 19(2) (1984), 299-323.

[9] R. S. Martin, Minimal positive harmonic functions, Trans. Amer. Math. Soc. 49 (1941), 137-172.

[10] H. P. McKean and D. Sullivan, Brownian motion and harmonic functions on the class surface of the thrice punctured sphere, Adv. Math. 51 (1984), 203-211.

[11] W. H. Meeks III, Global problems in classical minimal surface theory, in ed. D. Hoffman, Global Theory of Minimal Surfaces, American Mathematical Society, Providence, RI, for the Clay Mathematics Institute, Cambridge, MA, 2005, 453-470.

[12] W. H. Meeks III, J. Pérez, and A. Ros, Embedded minimal surfaces: removable singularities, local pictures and parking garage structures, the dynamics of dilation invariant collections and the characterization of examples of quadratic curvature decay. Preprint.

[13] W. H. Meeks III, J. Pérez, and A. Ros, The geometry of minimal surfaces of finite genus IV; Calabi-Yau conjectures. Preprint.

[14] W. H. Meeks III, J. Pérez, and A. Ros, The geometry of minimal surfaces of finite genus II; nonexistence of one limit end examples, Invent. Math. 158 (2004), 323-341.

[15] W. H. Meeks III and H. Rosenberg, Maximum principles at infinity with applications to minimal and constant mean curvature surfaces, Preprint.

[16] W. H. Meeks III and H. Rosenberg, The minimal lamination closure theorem, Duke Math. J. 133(3) (2006) 467-497.

[17] W. H. Meeks III and H. Rosenberg, The global theory of doubly periodic minimal surfaces, Invent. Math. 97 (1989), 351-379, MR1001845, Zbl 676.53068 . 
[18] W. H. Meeks III and H. Rosenberg, The geometry of periodic minimal surfaces, Comment. Math. Helvetici 68 (1993), 538-578, MR1241472, Zbl 807.53049.

[19] W. H. Meeks III and H. Rosenberg, The uniqueness of the helicoid and the asymptotic geometry of properly embedded minimal surfaces with finite topology, Ann. Math. 161 (2005), 723-754.

[20] S. Morales, On the existence of a proper minimal surface in $\mathbb{R}^{3}$ with the conformal type of a disk, GAFA 13(6) (2003), 1281-1301.

[21] A. V. Pogorelov, On the stability of minimal surfaces, Soviet Math. Dokl. 24 (1981), 274-276.

[22] A. Ros, The Gauss map of minimal surfaces, in Differential Geometry, Valencia 2001, Proceedings of the Conference in Honour of Antonio M. Naveira, World Scientific, 2002, 235-252.

[23] L. Sario and M. Nakai, Classification theory of Riemann surfaces, Grundlehren der Mathematischen Wissenschaften 164, Springer-Verlag, 1970.

[24] M. Soret, Minimal surfaces with bounded curvature in Euclidean space, Commun. Anal. Geom. 9(5) (2001), 921-950.

[25] S. T. Yau. A general Schwarz lemma for Kähler manifolds, Amer. J. Math. 100(1) (1975), 197-203.

Department of Mathematics

UNIVERSITY OF MASSACHUSETTS

AMHERST

MA 01003

USA

E-mail address: bill@math.umass.edu

Department of Geometry and Topology

UNIVERSITY OF GRANADA

GRANADA 18071

SPAIN

E-mail address: jperez@ugr.es

E-mail address: aros@ugr.es

Received March 10, 2006 
